\title{
Mowat-Wilson Syndrome: The First Clinical and Molecular Report of an Indonesian Patient
}

\author{
Farmaditya E. P. Mundhofir, ${ }^{1,2}$ Helger G. Yntema, ${ }^{2}$ Ineke van der Burgt, ${ }^{2}$ \\ Ben C. J. Hamel, ${ }^{2}$ Sultana M. H. Faradz, ${ }^{1}$ and Bregje W. M. van Bon ${ }^{2}$ \\ ${ }^{1}$ Division of Human Genetics, Center for Biomedical Research (CEBIOR), Faculty of Medicine, Diponegoro University GSG, \\ 2nd Floor Jl. Dr. Sutomo 14, Semarang, Indonesia \\ ${ }^{2}$ Department of Human Genetics, Radboud University Nijmegen Medical Centre, P.O. Box 9101, \\ 6500 HB Nijmegen, The Netherlands
}

Correspondence should be addressed to Bregje W. M. van Bon, b.vanbon@gen.umcn.nl

Received 20 October 2012; Accepted 6 November 2012

Academic Editors: S. Chappell, R. Perry, and M. Suri

Copyright (C 2012 Farmaditya E. P. Mundhofir et al. This is an open access article distributed under the Creative Commons Attribution License, which permits unrestricted use, distribution, and reproduction in any medium, provided the original work is properly cited.

\begin{abstract}
Mowat-Wilson syndrome (OMIM 235730) is a genetic condition characterized by moderate-to-severe intellectual disability, a recognizable facial phenotype, and multiple congenital anomalies. The striking facial phenotype in addition to other features such as severely impaired speech, hypotonia, microcephaly, short stature, seizures, corpus callosum agenesis, congenital heart defects, hypospadias, and Hirschsprung disease are particularly important clues for the initial clinical diagnosis. All molecularly confirmed cases with typical MWS have a heterozygous loss-of-function mutation in the zinc finger E-box protein 2 (ZEB2) gene, also called SIP1 (Smad-interacting protein 1) and ZFHX1B, suggesting that haploinsufficiency is the main pathological mechanism. Approximately $80 \%$ of mutations are nonsense and frameshift mutations (small insertions or deletions). About half of these mutations are located in exon eight. Here, we report the first Indonesian patient with Mowat-Wilson syndrome confirmed by molecular analysis.
\end{abstract}

\section{Introduction}

Mowat-Wilson syndrome (MWS; OMIM 235730) is a rare genetic condition described by Mowat et al. in 1998, who reported a series of six children with intellectual disability (ID), striking facial features, and variable multiple congenital anomalies (MCA) [1]. All molecularly confirmed cases with typical MWS have a heterozygous loss of function mutation in the zinc finger E-box protein 2 (ZEB2) gene, also called SIP1 (Smad-interacting protein 1) and ZFHX1B [2]. To date, about 200 molecularly proven MWS cases with over 100 different ZEB2 mutations have been reported [3].

The facial features are the most important diagnostic clue for the initial clinical diagnosis and provide a hallmark for ZEB2 mutation analysis [4]. Establishing a molecular diagnosis is important for the patients and their families as it allows reliable genetic counseling for their families and a better clinical management of the patients. Here, we report the first Indonesian patient with molecularly confirmed MWS.

\section{Case Presentation}

The patient was a nineteen-year-old male with severe ID. He was the third son of nonconsanguineous, healthy, Javanese parents and family history was unremarkable. The patient was born at term after an uneventful pregnancy with a weight of $3200 \mathrm{~g}$ (25th centile) and length $50 \mathrm{~cm}$ (50th centile). He showed hypotonia and delayed developmental milestones. He started to sit at 20 months of age. At two years of age, he developed recurrent generalized seizures and was commenced on valproic acid, which brought his epilepsy under control. He started to walk at four years of age and spoke his first words at the age of five years. He had recurrent otitis media. Speech consisted of only a few words and he often communicated using sign language. He showed happy behavior with frequent smiling. In addition, he showed repetitive hand movements. On physical examination, his weight was $45 \mathrm{~kg}$ ( $<3 \mathrm{rd}$ centile), height $161 \mathrm{~cm}$ ( $<3$ rd centile), and head circumference $53 \mathrm{~cm}(<3$ rd centile). 


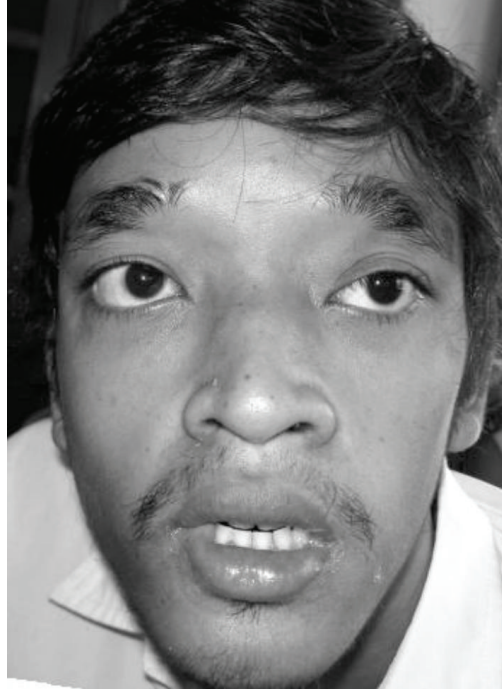

(a)

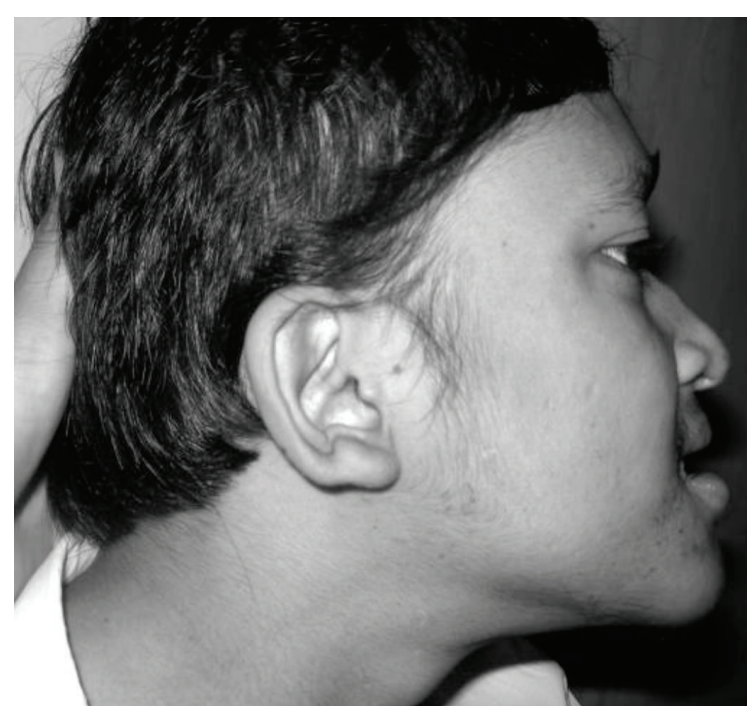

(b)

FIGURE 1: Photograph of Indonesian patient with Mowat-Wilson syndrome. Our patient in his 19 years of age showed striking facial gestalts of MWS such as large eyebrows with medial flaring (a) and uplifted ear lobules (b). Other dysmorphisms such as long face, deep-set eyes, upward slanting palpebral fissures, hypertelorism, strabismus, saddle nose with prominent rounded nasal tip, prominent columella, low-set and posteriorly rotated ears, prominent and triangular pointed chin, small mouth, full lips, and prognathia are noted.

Facial dysmorphisms included a long face, deep-set eyes, large eyebrows with medial flaring, hypertelorism, strabismus, saddle nose with prominent rounded nasal tip, prominent columella, low-set and posteriorly rotated ears, uplifted ear lobules, a prominent narrow pointed chin, a small mouth, and prognathism (Figure 1). In addition, he had tapered and slender fingers, prominent interphalangeal joints, and bilateral pes planus. Generalized hypotonia and hyperreflexia were observed. Heart auscultation was normal.

The individual was part of a larger series of 527 Indonesian individuals with ID from schools and institutions, whose conventional karyotyping, FMR1 gene analysis, and subtelomeric MLPA were normal [5]. Based on the clinical features, MWS was suspected. Therefore, molecular analysis of the ZEB2 gene was warranted. Sanger sequencing of all coding exons and surrounding splice sites of the ZEB2 gene was performed as described below. The genomic DNA reference sequence was NM_014795.2. PCR of exon eight was performed using primers CTTTACTTGGGTTTCCCACC (forward) and GGGGCTTGTCATTCCTT (reverse). One hundred nanograms of DNA solution $(1 \mu \mathrm{L})$ were added into PCR mixture, which contained 7.6 $\mu \mathrm{L}$ of 360 PCR master mix (Applied Biosystem), $0.5 \mu \mathrm{L}$ of primers working solution, and $6 \mu \mathrm{L}$ of $\mathrm{H}_{2} \mathrm{O}$. Amplification was performed using PCR System 9700 (Applied Biosystem) with the following protocol. PCR was initiated by $10^{\prime}$ denaturation at $95^{\circ} \mathrm{C}$, followed by $35 \mathrm{PCR}$ cycles $\left(30^{\prime \prime} 95^{\circ} \mathrm{C}, 30^{\prime \prime} 60^{\circ} \mathrm{C}, 60^{\prime \prime} 72^{\circ} \mathrm{C}\right)$ and $7^{\prime}$ final elongation at $72^{\circ} \mathrm{C}$. The result was analyzed on ABI 3730 analyzer (Applied Biosystem). Sequence result was compared to published reference sequence (rs148709333) using SEQPilot software version 3.2.1.0 (JSI medical system). In exon eight, a nonsense mutation has been detected, changing a TAC codon (coding for a tyrosine) into a TAG stopcodon; c.1965C > G (p. (Tyr652*)) (nomenclature according

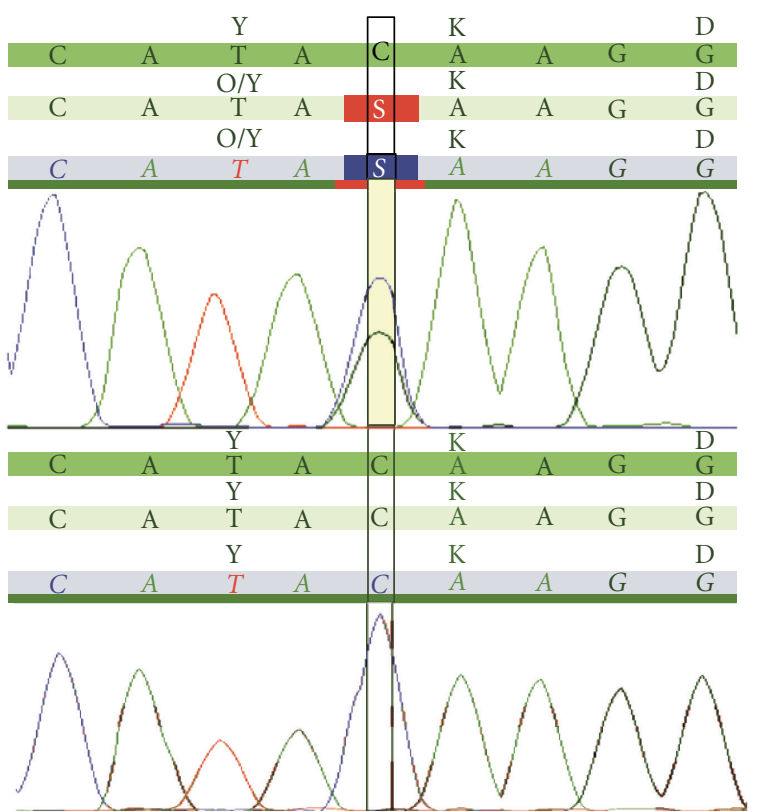

FIGURE 2: Electropherogram of molecular analysis in the patient sample. The upper panel shows the heterozygous c.1965C $>\mathrm{G}$ (p.Tyr652X) mutation and the lower panel shows the wild type (control). The "S" on the electropherogram represents the $\mathrm{C} / \mathrm{G}$ heterozygote.

to the HGVS guidelines; http://www.hgvs.org/mutnomen/) (Figure 2). To our knowledge, this mutation has not been reported before.

\section{Discussion}

This is the first report of an Indonesian individual with MWS confirmed by molecular genetic testing. Although nonsense 
TABLE 1: Clinical features of our patient compared to those in published cases of MWS with proven ZEB2 mutations.

\begin{tabular}{lcc}
\hline Clinical features & Our patient & $\begin{array}{c}\text { Mowat-Wilson } \\
\text { syndrome* }\end{array}$ \\
\hline ZEB2 mutations & + & $100 \%$ \\
Intellectual disability & + & $100 \%$ \\
Typical facial gestalt & + & $97 \%$ \\
Microcephaly & + & $81 \%$ \\
Seizures & + & $73 \%$ \\
HSCR & $-* *$ & $57 \%$ \\
CHD & $-* *$ & $52 \%$ \\
Hypospadias & - & $52 \%$ \\
Short stature & + & $46 \%$ \\
Hypoplasia or agenesis of CCA & $\mathrm{NT}$ & $43 \%$ \\
Cryptorchidism & - & $36 \%$ \\
Constipation & - & $26 \%$ \\
Pyloric stenosis & - & $4.7 \%$ \\
Eye anomalies & - & $4.1 \%$ \\
Cleft palate & - & $2.9 \%$ \\
\hline
\end{tabular}

*Adapted from Garavelli and Mainardi (2007) [4].

**Symptoms not observed although the gold standard diagnosis has not been performed.

NT: Not Tested, HSCR: Hirschprung Disease, CHD: Congenital Heart Defect, CCA: Corpus Callosum.

mutations account for more than $40 \%$ of known ZEB2 mutations and approximately $50 \%$ of these are localized in exon eight [6], the particular mutation detected in the patient described in this paper (c.1965C>G; p.Tyr652*) has not been reported before.

Most clinical features of our patient, who had severe ID, a distinct facial gestalt, microcephaly, and seizures, are consistent with those described in the literature (Table 1). Brain imaging and echocardiography could not be performed since he is living in the country with minimal health facilities. Symptoms of Hirschsprung disease (HSCR) such as constipation, dysphagia, and poor appetite were not reported in our patient, but the prevalence of these symptoms in other publications ranged from the majority of individuals $[1,2]$ to $50 \%$ of cases $[4,6]$. Early diagnosis, intervention, and targeted management are necessary for a better health and life quality of individuals with MWS. However, as this syndrome is rare and recently described, the knowledge of the clinical complications and natural history is still developing [7].

In summary, we report the first Indonesian MWS case with a novel ZEB2 mutation. Our patient showed similar dysmorphism to previously reported cases, although several major associated features were not present such as HSCR, congenital heart defect (CHD), and hypospadia. Despite the availability of molecular diagnostic tests in several parts of the world, the recognition of clinically well-defined syndromes will remain very important in countries with limited diagnostic facilities such as Indonesia. The publication of cases with recognizable facial features is therefore of great importance in order to make local pediatricians aware of rare conditions like Mowat-Wilson syndrome, allowing more clinical diagnoses in the future.

\section{Conflict of Interests}

The authors have no conflict of interests to declare.

\section{Acknowledgments}

This research is partly funded by the Overseas study Scholarship (Beasiswa Luar Negeri) of the Directorate General of Higher Education (DGHE), Ministry of National Education and Culture Republic of Indonesia, and the $\mathrm{PhD}$ Fellowship program of Radboud University Nijmegen (RU Fellowship). The authors thank the family of the patient for their cooperation and permission to publish this paper. They also thank laboratory staff at Department of Human Genetics, RUNMC, The Netherlands and CEBIOR, FMDU, Semarang Indonesia, in particular Dr. Tri Indah Winarni, Willy Nillesen, and Martine van Zweeden.

\section{References}

[1] D. R. Mowat, G. D. H. Croaker, D. T. Cass et al., "Hirschsprung disease, microcephaly, mental retardation, and characteristic facial features: delineation of a new syndrome and identification of a locus at chromosome 2q22-q23," Journal of Medical Genetics, vol. 35, no. 8, pp. 617-623, 1998.

[2] N. Wakamatsu, Y. Yamada, K. Yamada et al., "Mutations in SIP1, encoding Smad interacting protein-1, cause a form of Hirschsprung disease," Nature Genetics, vol. 27, no. 4, pp. 369370, 2001.

[3] E. Evans, S. Einfeld, D. Mowat et al., "The behavioral phenotype of Mowat-Wilson syndrome," American Journal of Medical Genetics A, vol. 158, no. 2, pp. 358-366, 2012.

[4] L. Garavelli and P. C. Mainardi, "Mowat-Wilson syndrome," Orphanet Journal of Rare Diseases, vol. 2, no. 1, article 42, 2007.

[5] F. E. Mundhofir, T. I. Winarni, B. W. van Bon et al., "A cytogenetic study in a large population of intellectually disabled Indonesians," Genetic Testing and Molecular Biomarkers, vol. 16, no. 5, pp. 412-417, 2012.

[6] F. Dastot-Le Moal, M. Wilson, D. Mowat, N. Collot, F. Niel, and M. Goossens, "ZFHX1B mutations in patients with MowatWilson syndrome," Human Mutation, vol. 28, no. 4, pp. 313321, 2007.

[7] D. R. Mowat and M. J. Wilson, "Mowat-Wilson syndrome," in Management of Genetic Syndromes, S. B. Cassidy and J. E. Allanson, Eds., pp. 517-529, John Wiley and Sons, New York, NY, USA, 2010. 


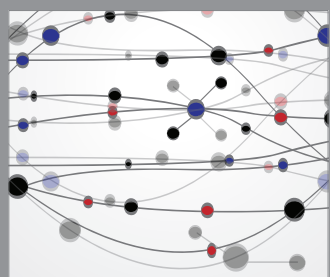

The Scientific World Journal
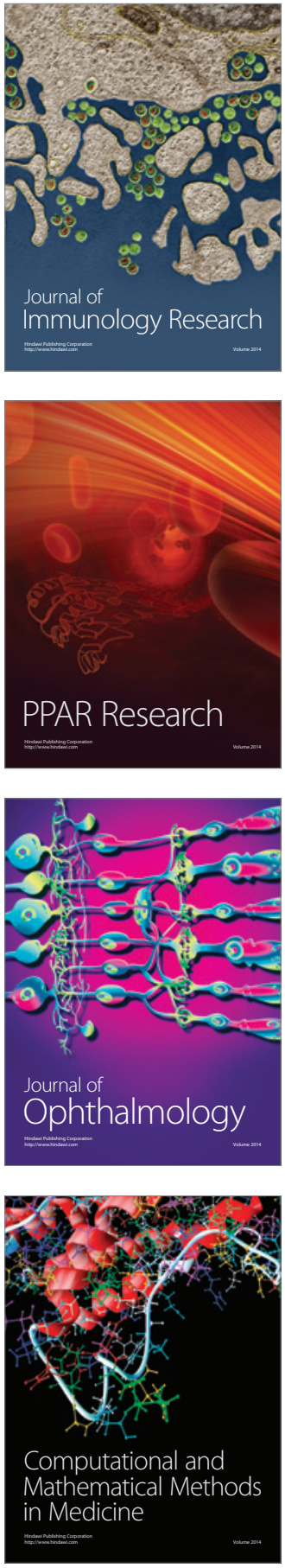

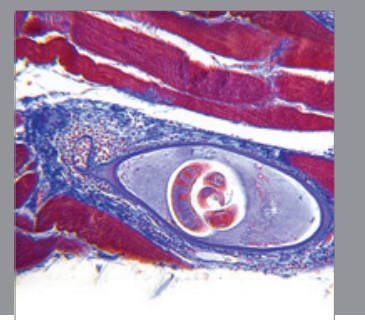

Gastroenterology

Research and Practice
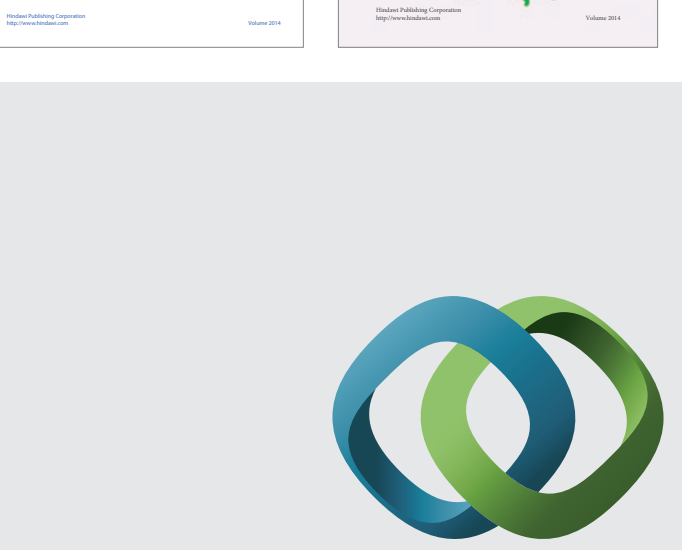

\section{Hindawi}

Submit your manuscripts at

http://www.hindawi.com
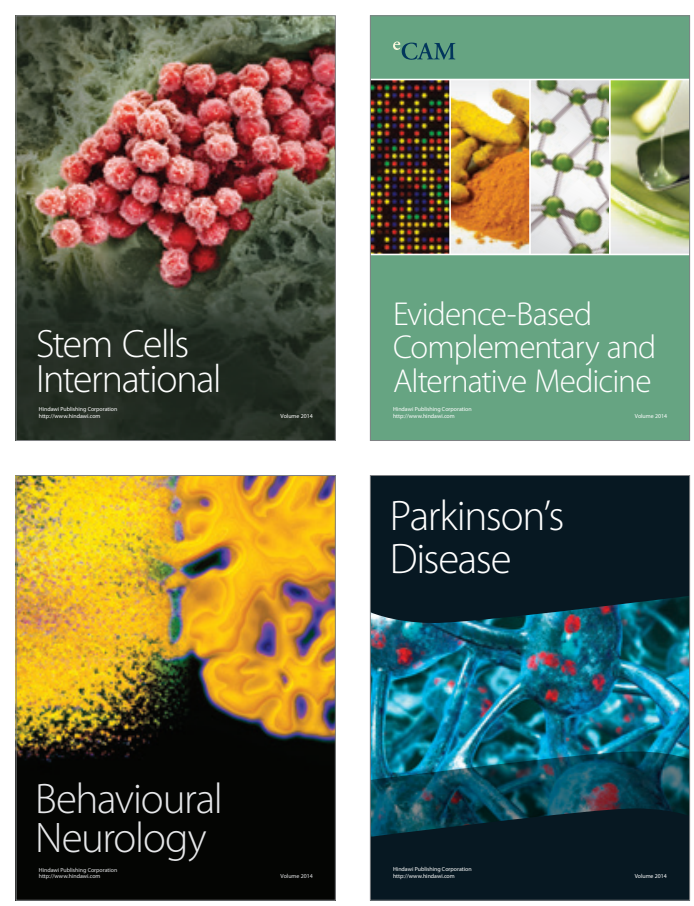

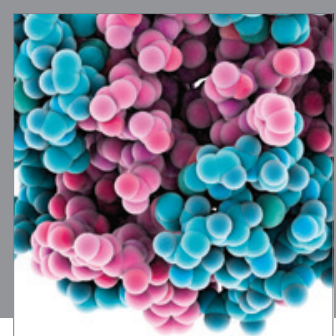

Journal of
Diabetes Research

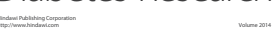

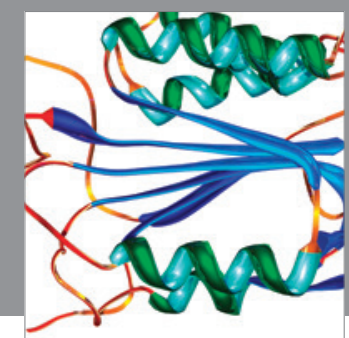

Disease Markers
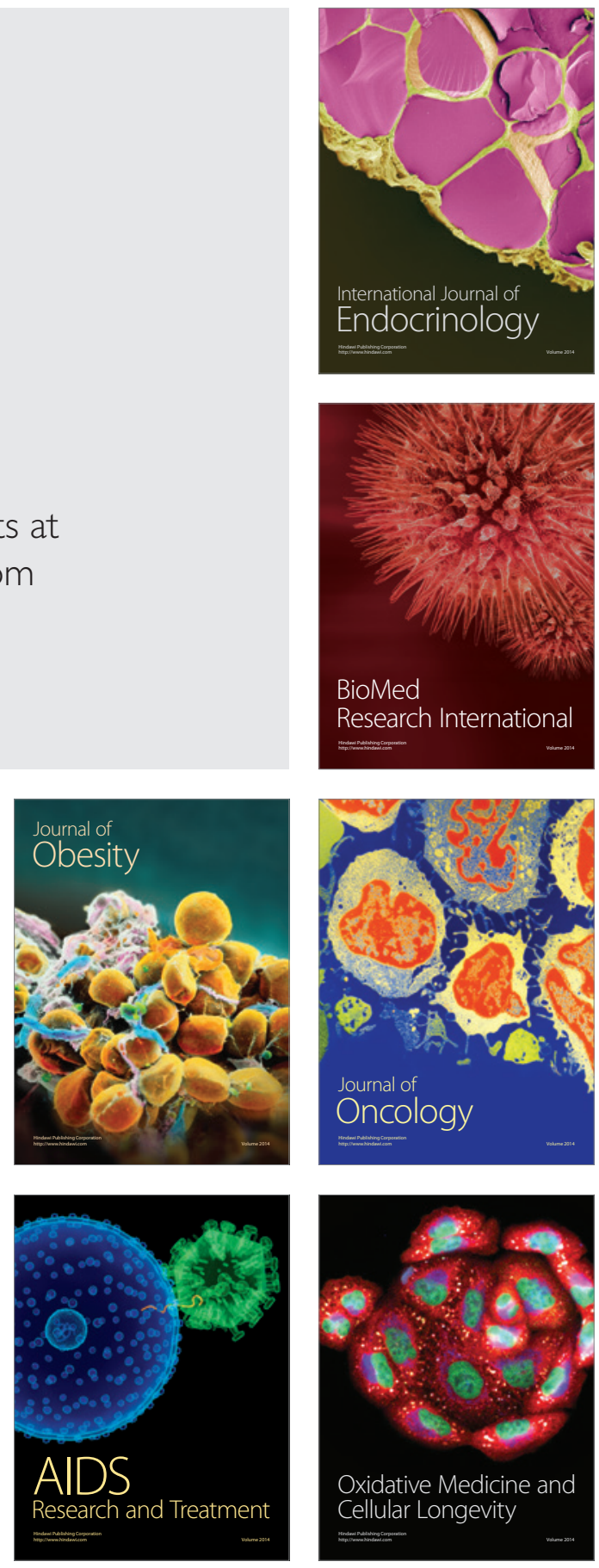